\title{
BCG 1948 - 2014: ¿LA MISMA CEPA?
}

\section{BCG 1948 - 2014: is this still the same strain?}

\author{
Dr. Rodolfo Villena Martínez \\ Pediatra Infectólogo \\ Unidad de Infectología - Hospital de niños Dr Exequiel González Cortés \\ Profesor Asistente, Facultad de Medicina \\ Universidad de Chile
}

\begin{abstract}
BCG 1948 - 2014: IS THIS STILL THE SAME STRAIN?
Tuberculosis remains a public health problem and its control is a global health priority. One of the most successful tools to control infectious diseases has been the use of vaccines. Bacille Calmette-Guérin is one of the oldest available vaccines used today, the only licensed against tuberculosis. It has been administered to billions of people, as part of national immunization programs around the world. Immunological mechanisms by which it induces protection are not fully understood, but a role in the innate immune system maturation and the activation of T CD $4+$ and CD $8+$, have been considered. Its effectiveness in terms of pulmonary tuberculosis is variable and controversial but highly cost - efficient to control tuberculous meningitis and miliary dissemination. However, it has not been enough to solve the global problem of tuberculosis, especially in countries with high rates, so that scientific development aiming at getting a new vaccine remains active.
\end{abstract}

Key words: BCG, tuberculosis, vaccines.

\section{RESUMEN}

La tuberculosis sigue siendo un problema de salud pública mundial y su control es una prioridad de salud global. Una de las herramientas más exitosas que se han utilizado para controlar enfermedades infecciosas ha sido el uso de vacunas. El bacilo de Calmette-Guérin es una de las vacunas más antiguas disponibles utilizadas en la actualidad, la única licenciada contra tuberculosis. Ha sido administrada en miles de millones de personas, siendo parte de distintos programas de vacunación nacionales en el mundo. Los mecanismos inmunológicos por los cuales induce protección aún no son completamente comprendidos, planteándose un rol sobre la maduración del sistema inmune innato y activación de células T CD4+ y CD8+. Su eficacia respecto de tuberculosis pulmonar es variable y controvertida, pero altamente costo eficiente para controlar meningitis tuberculosa y diseminación miliar, sin embargo no ha sido suficiente para resolver el problema global de la tuberculosis, especialmente en los países con endemias más altas, por lo que el desarrollo científico en miras de obtener una nueva vacuna se mantiene vigente.

Palabras clave: BCG, tuberculosis, vacunas.

\section{INTRODUCCIÓN}

La tuberculosis (TB) es una enfermedad que ha afectado a los humanos desde miles de años atrás sin existir países del mundo que sean considerados libres de ella (1). Actualmente causa cerca de 9 millones de infecciones y 1,5 millones de muertes cada año (2), manteniéndose como un problema de salud pública mundial que requiere vigilancia activa y la participación de múltiples sectores para poder contenerla. En 1882 Robert Koch identificó al bacilo que producía la TB

\section{Correspondencia:}

Dr. Rodolfo Villena

Hospital Dr Exequiel González Cortés

Barros Luco 3344 - San Miguel - Santiago de Chile

Correo electrónico: rodolfo.villena@redsalud.gov.cl humana, reconociéndola como una enfermedad infecciosa, Io que incentivó el desarrollo de distintas estrategias para tratar de controlarla, como los sanatorios, la pasteurización de los productos lácteos, las drogas antiTB y la creación de vacunas BCG. Sin embargo aún no hemos logrado solucionar el problema de la TB en ningún país del mundo $(1,3,4)$.

\section{PASADO Y PRESENTE DE BCG}

BCG (bacilo de Calmette-Guérin) es una vacuna viva derivada de Mycobacterium bovis, la única licenciada contra TB en el mundo. Los estudios de estos dos científicos franceses en el Instituto Pasteur se iniciaron en 1908, subcultivando la cepa cada 3 semanas por 13 años, totalizando 231 ciclos, hasta obtener el producto final. Posteriormente transfirieron algunas de ellas a docenas de laboratorios a nivel mundial, cada uno de 
Ios cuales realizó procesos propios de subcultivos en diferentes condiciones, siendo las cepas originales eliminadas. De esta manera se generaron varias cepas con cambios fenotípicos entre ellas y pérdida de factores de virulencia, los cuales no están bien precisados (3). Por este motivo se dice que BCG no es una vacuna única, sino que corresponde a una familia de vacunas, siendo hoy en día el grupo de vacunas más antiguas disponibles que aún se están utilizando, conformando parte de los programas nacionales de inmunización (PNI) de casi todos los países en el mundo, en distintas etapas de su desarrollo económico e histórico, excepto en Estados Unidos y Holanda, estimándose que más de 4 mil millones de personas las han recibido $(1,3)$.

En 1921 se vacunó al primer humano con BCG y en 1928 la Liga de las Naciones la adoptó como vacuna. Entre 1929 y 1930 ocurrió el desastre de Lübeck, donde fallecieron 72 niños posterior a la contaminación con un bacilo virulento de la preparación oral de BCG que se utilizaba en ese entonces. En 1947 se introduce la técnica inyectable que se ocupa en la actualidad y en 1948, el primer congreso internacional sobre BCG determinó que era efectiva y segura contra TB, a pesar de la falta de estudios apropiados para sostener esta afirmación, extendiéndose su vacunación a nivel mundial, lo cual fue seguido por campañas apoyadas por OMS y UNICEF, con lo que miles de millones de personas lograron ser vacunadas. En 1956 la OMS solicitó liofilizar los lotes disponibles para preservar las cepas respecto de las originales, con el objetivo de estandarizar su producción y características vacunales $(1,3)$.

En Chile el uso de esta vacuna se remonta a 1927, en las primeras vacunaciones orales en recién nacidos, legalizada con el Decreto 1841 que incorporó la vacuna al "Programa de Lucha contra la Tuberculosis". Fue suspendida en 1930 por la tragedia de Lübeck, reincorporándose en 1947 con técnica intradérmica, programándose metas de coberturas del 95\% (5). De esta manera el 2011 habríamos tenido 26.560 defunciones por TB, en caso de no haber incorporado la vacuna, en lugar de las 236 muertes reales observadas ese año (5). Claramente estos resultados son multifactoriales y fruto de un programa de salud pública consistente y bien organizado que ha permitido a Chile transitar hacia la eliminación de esta enfermedad, reduciendo su incidencia desde 41,3 por 100.000 habitantes en 1990 a 13,6 en el año 2013 (6). La cobertura de BCG durante el 2012 en siete de las 15 regiones estaba bajo el 95\% (6), ascendiendo hasta $97,6 \%$ el 2014 , usando como denominador los recién nacidos vivos, lo que representa una mejora respecto del 2013 (93\%).

Su presentación es en frascos multidosis (10 ó 20 dosis), duran 4 horas una vez abierto el envase, no poseen timerosal ni otros preservantes, y sus precios por dosis oscilan entre \$84 y $\$ 282$ (5).

\section{INMUNOGENICIDAD Y CORRELATOS DE PROTECCIÓN}

Estudios de laboratorio han demostrado que existen algunas cepas denominadas fuertes (Pasteur 1173 P2, Danesa 1331) y otras débiles (Glaxo 1077 y Tokio 172). Los patrones de fragmentos de restricción mayores originados a través de la digestión del DNA de las vacunas BCG son distintos y pueden ayudar para identificarlas. Las cepas fuertes poseen mayor inmunogenicidad, hipersensibilidad cutánea, lesiones granulomatosas y probablemente mayor protección contra TB (3). Entre los cambios genotípicos más relevantes está la pérdida de la región RD1, la cual codifica para 9 proteínas de M tuberculosis que no se encuentran en BCG. Esta deleción Ilevó a la pérdida de los antígenos ESAT y CFP10, manteniendo otros como el Ag85, MPB70, y el micósido $B$, este último se modifica según métodos de producción de la vacuna $(1,3,7-9)$.

Los eventos inmunológicos que ocurren en el hospedero posterior a la vacunación con BCG o en respuesta a la infección por $M$ tuberculosis no son conocidos en profundidad, pero en modelos animales se ha visto que los linfocitos $T$ CD4+ jugarían un papel protagónico, generando células $T$ de memoria central, pero existiría una incapacidad de la vacuna de inducirlas por un largo periodo de tiempo, por lo que se perdería la protección dentro de 15 - 20 años posterior a la vacunación $(3,10)$. De esta manera no se dispone de correlato de protección confiable, desconociéndose el nivel de protección que otorga la vacuna y la enfermedad en cada individuo. Estudios en humanos sugieren que el IFN-y no necesariamente se correlaciona con protección, planteándose medir complejos cuantitativos, a través de una combinación de citoquinas $(7,10,11)$. Todavía existen antígenos lipídicos y proteínas del M. tuberculosis cuya función es desconocida, presentes en distintas fases de crecimiento, que podrían gatillar la respuesta inmune innata, a través de los tolllike receptors 2 y 6 , pudiendo ser las claves para comprender la incapacidad para eliminar el bacilo que se observa en algunos pacientes.

La inmunidad necesaria para responder a una infección por M. tuberculosis dependerá de la fase de ella y el equilibrio que exista entre sus componentes. Inicialmente la inmunidad innata ingiere y mata a la bacteria a través de los macrófagos, con un importante rol de los neutrófilos, lo que serviría para entrenar al sistema inmune innato a través de inducción de citoquinas proinflamatorias, lo que incluso tendría implicancias en disminuir la mortalidad por sepsis de cualquier causa en prematuros vacunados con BCG (12). Posteriormente linfocitos T CD4+, a través de citoquinas como interleukina 2 e interferón gamma (IFN-y), activan a fagocitos para que eliminen bacilos. Los linfocitos CD8+ estimulan la necrosis y destrucción celular de los fagocitos que intentan eliminar al bacilo, sin conocerse de manera completa cuáles son los procesos por los que atraviesa el macrófago para evitar los escapes de la enfermedad (3). Esta respuesta de linfocitos CD8+ es lo que determina la respuesta de hipersensibilidad tardía (10), lo que todavía se utiliza como evidencia de infección o un signo de adecuada respuesta a la vacuna $B C G$, a través de la prueba de tuberculina, sin embargo sus magnitudes de reacción no se correlacionan necesariamente con nivel de protección. La aplicación de proteinómica junto al desarrollo del conocimiento del genoma han acelerado la identificación de proteinas bacterianas y su uso como correlatos de protección y sus aplicaciones diagnósticas, por lo que se espera que en los próximos años este escenario cambie y se disponga de herramientas más concretas para entender la protección conferida tanto por la enfermedad como por las vacunas BCG (7). 


\section{CICATRIZ VACUNAL Y PPD}

La vacunación con BCG produce una cicatriz, dos a tres semanas después de su administración apropiada. Se desarrolla una pápula en el sitio de administración, que crece hasta 8 $\mathrm{mm}$ en la semana 5 , hasta que se ulcera y posteriormente cura espontáneamente en la semana 6 a 12, dejando una cicatriz permanente despigmentada plana o deprimida. Esta cicatriz puede tardar hasta 6 meses o más en desarrollarse e incluso pudiese ser negativa en un $10-15 \%$ de los casos cuando es administrada al momento de nacer. También puede ocurrir en un $10 \%$ de los casos que la lesión desaparezca en el tiempo $(13,14)$. Estos dos escenarios no se correlacionan con ausencia de inmunidad (8).

Si la técnica de vacunación se aplica correctamente, con una vacuna conservada en buenas condiciones, los test tuberculínicos tres meses después deben ser positivos en el $95 \%$ de los casos. Desafortunadamente no existe un método confiable que pueda distinguir entre reacción cutánea inducida por vacunación por BCG o por infección con M tuberculosis. La proporción de sujetos vacunados con BCG pueden presentar un amplio espectro de reacciones tuberculínicas, variando desde 0 a $90 \%$ de los casos. El tamaño de esta reacción cutánea varía según dosis, cepa, ruta de administración, edad del paciente, estado nutricional, tiempo desde la vacunación e inclusive si es que la dosis es reiterada $(3,15,16)$. La variación de la prueba de tuberculina desde negativo $(<5 \mathrm{~mm})$ a positivo $(>10-15 \mathrm{~mm})$ es útil en sujetos no vacunados de países con baja endemia. En países con micobacterias ambientales $\mathrm{y} / 0$ altas endemias la interpretación de estas pruebas son dificultosas porque no diferencian entre exposición previa 0 TB activa $(1,8,17)$.

\section{ESTUDIOS DE EFICACIA}

Las vacunas BCG proveen una robusta protección contra mortalidad por meningitis (64\%) y TB miliar (78\%) en la infancia, con una protección global del 50\%, sin embargo su protección contra TB en adolescentes y adultos, donde se concentran los casos de TB, es altamente variable, dependiendo de los estudios, poblaciones y coberturas analizadas, por lo que la inmunogenicidad y eficacia son objeto de controversia $(1,7,18,19)$. Incluso en las áreas de alto riesgo de TB, el uso de BCG no debiera ser considerado el principal método de control de protección, debido a que su eficacia es desconocida, no protege a los no vacunados y el test tuberculina puede ser confundente para aceptarlo como correlato de protección.

Estimaciones de eficacia de BCG en la prevención de TB pulmonar varían desde $0 \%$ en Chingleput, India (20) hasta $80 \%$ en el Medical Research Council en el Reino Unido (21). Eficacia contra enfermedad primaria progresiva ha sido reportada de manera exitosa en los países con vacunación neonatal, persistiendo tras 10 años de la vacunación (3). Estudios en poblaciones originarias en Estados Unidos demostraron una eficacia cercana al $50 \%$ en protección contra TB pulmonar en adultos mayors de 50 años (22). Se desconoce si la latitud afecta la eficacia, pero se plantea que mientras más cerca de la línea ecuatorial esta pudiese disminuir por interferencia inmunológica con micobacterias ambientales $(1,18,23)$. Otro de los problemas para determinar la eficacia y efectividad de las vacunas es que la TB infantil es más difícil de diagnosticar y confirmar que en adultos, pudiendo las formas moderadas no haber sido reconocidas en estos estudios, además de los factores socio culturales, económicos y medio ambientales $(1,24)$.

\section{INDICACIONES}

La OMS y la unión internacional contra la TB y la enfermedad pulmonar sugieren los siguientes criterios para decidir remover la vacunación universal con BCG: si es que existe un buen sistema de notificación con tasas < 5 por 100000 habitantes 0 el promedio anual de notificación de meningitis TB en $<5$ años de edad $<1$ por 10 millones de habitantes en relación a los 5 años previos, 0 si el promedio de riesgo anual de TB es $<0,1 \%$. A medida que estas situaciones se van presentando los países han ido orientándose hacia vacunación en poblaciones selectivas, descontinuando la vacunación universal $(1,3)$. La cepa, esquema, dosis, ruta de administración ni edad óptimas han sido establecidas de manera categórica para el uso de vacunas BCG (3). La OMS recomienda dosis única de 0,05 $\mathrm{ml}$ en los recién nacidos, estrictamente por vía intradérmica, correspondiendo a la mitad de la dosis recomendada en sujetos mayores, principalmente para países en vías de desarrollo con alta prevalencia de TB. Esto ha tenido un impacto económico y de mortalidad a corto plazo, sin contribuir de manera importante al control de la TB (1).

La experiencia muestra que la asociación de BCG con otras vacunas no produce, por lo general, aumento de la reactogenididad ni modificaciones en la respuesta vacunal. Ninguna otra vacuna debe ser usada en la extremidad donde se puso BCG por al menos 3 meses, debido al riesgo de linfadenitis existente. En caso de querer administrar otra vacuna viva atenuada inyectable, deben espaciarse por al menos 4 semanas (1). Hoy en día la única indicación en mayores de 16 años es en sujetos con alto riesgo de exposición, como trabajadores de laboratorio y personal de salud. Este último escenario se plantea solo en condiciones de alto riesgo de transmisión de TB multi resistente, en los cuales las estrategias habituales de control y profilaxis quimioterápicas han fallado (3). No hay evidencias de que la repetición de la vacunación sea necesaria, a pesar de la pérdida de inmunidad.

Las administraciones orales actualmente no son recomendadas. La mayoría de los países utilizan rutas intradérmicas, para medir de manera precisa la dosis administrada. La ubicación más habitual es a nivel del deltoides, sin embargo puede variar en distintos países. La vía intradérmica produce mayor cantidad de casos de úlceras y adenitis. La vía subcutánea tiene el riesgo de abscesos y cicatrices con retracción en el sitio de administración. Existe una técnica con dispositivo de agujas múltiples que posee menos efectos secundarios. Se desconoce si es que existe alguna diferencia entre las técnicas de administración y la protección contra TB $(1,3,13)$.

La cicatriz BCG no traduce inmunidad y su ausencia no se correlaciona con falta de protección $(25,26)$, sin embargo 
algunos países revacunan frente a ausencia de ella $(1,3,25)$. En Chile en caso de niños que no presenten cicatriz tras la inmunización solo se considera imprescindible asegurar con algún medio verificable de que esté vacunado, no requiriéndose en estos casos realizar PPD ni revacunar. Tampoco se recomienda vacunar a mayores de 4 años, independiente de su procedencia, ni a menores de 4 años procedentes del extranjero no vacunados con BCG que tienen planeado estar menos de 6 meses en nuestro país, excepto que residan en zonas de mayor incidencia de TB, como Arica y Parinacota y Tarapacá (25).

\section{EFECTOS SECUNDARIOS}

La vacunación con BCG es segura, sus complicaciones son raras, pero varían según destrezas y método de administración; dosis y tipo de vacuna; y edad e inmunidad del paciente, siendo mejor tolerada en menores de 6 meses, con tasas de reacciones locales y abscesos más altas en mujeres y adultos $(1,3,13)$. Las reacciones locales importantes, abscesos y úlceras extensas, se presentan en 1 por 1000 vacunados, principalmente en inmunodeficientes y suelen ser consecuencia de una inadecuada técnica de administración, con inyección demasiado profunda. Otros eventos adversos son adenitis inflamatoria y osteítis del recién nacido, describiéndose mayor asociación con las cepas BCG fuertes. La incidencia para diseminación fatal es de 0,19 - 1,56 por millón de dosis, casi exclusivamente en sujetos con severo compromiso de inmunidad celular. En situaciones de sobredosis aumenta el riesgo de reacciones adversas locales, linfadenitis supurativas e inclusive una cicatriz excesiva $(1,3,13)$.

\section{CONTRAINDICACIONES}

Corresponden a las generales de todas las vacunas; 0 cuando clínicamente existe enfermedad tuberculosa; personas con inmunodeficiencias congénitas 0 adquiridas; neonatos con menos de $2.000 \mathrm{~g}$ de peso; eritroblastosis fetal; neonatos en cuya casa existe un caso sospechoso o confirmado de TB, embarazo; pacientes con desnutrición grave; niños con enfermedades cutáneas generalizadas; $y / 0$ antecedentes de anafilaxia a algún componente de la vacuna $(1,25,27)$. La vacunación en prematuros podría provocar apneas y bradicardia, lo cual no es una contraindicación para vacunar. En el caso de ser hijo de madre VIH positiva debe consultarse al médico encargado del programa respectivo antes de su vacunación, describiéndose que su postergación hasta la 8 a semana de vida en niños no infectados, podría tener una mayor respuesta de células T BCGespecíficas (28).

\section{DESARROLLO DE NUEVAS VACUNAS BCG}

Desde 1930 se han considerado candidatos alternativos a M bovis para inmunizar contra TB, tales como bacilos tuberculosos muertos u otras micobacterias ambientales no virulentas, sin embargo no han sido suficientemente exitosas, probablemente por desconocimiento de mecanismos inmunológicos más profundos de la enfermedad en sus distintas fases, métodos diagnósticos clínicos y de laboratorio sin un gold standard adecuado 0 interferencia con micobacterias ambientales. Nuevas técnicas biotecnológicas y de genética molecular se han desarrollado para permitir avances en este aspecto, como vacunas de subunidades (mezcla de proteinas de $M$ tuberculosis); vacunas DNA; vacunas que utilizan vehículos virales; vacunas recombinantes BCG (inserción de genes foráneos o sobreexpresión de genes nativos); y auxótropos y mutantes (cepa virulenta atenuada mediante deleción genética de factores de virulencia, presentando perfil de seguridad y biodistribución semejante a BCG, con niveles altos de protección en estudios preclínicos) $(3,7,9,29,30)$.

Todos estos candidatos deben demostrar eficacia en las distintas fases de la TB, tales como enfermedad, infección, latencia y/o cura, además de ser evaluadas en el contexto de las zonas epidemiológicas donde existe coinfección con VIH. Las claves para lograr estos objetivos están relacionadas con la creatividad en los candidatos desarrollados por la investigación básica; descubrir correlatos de inmunidad y biomarcadores para evaluar respuesta a vacunas contra TB; cooperación para el desarrollo de estudios clínicos; estandarización de los procesos de producción; selección racional de los candidatos de vacunas contra TB y compromisos multisectoriales para lograr aceptación y financiamiento de las nuevas vacunas. $(7,11)$.

\section{COMENTARIO FINAL}

BCG es una familia de vacunas seguras, ampliamente utilizadas en el mundo, con efectividad sobre manifestaciones miliar y meningea, sin embargo no son suficientes para resolver el problema global de TB, por lo que requerimos mantener nuestras estrategias multisectoriales, homogeneizar la cobertura a lo largo de nuestro país, profundizar nuestro conocimiento en su respuesta inmune en las distintas etapas de la infección, en espera de que los candidatos vacunales demuestren resultados más promisorios, por lo que por el momento debemos seguir aplicando BCG acorde a la situación epidemiológica y las recomendaciones internacionales.

\section{El autor declara no presentar conflicto de intereses}

\section{REFERENCIAS}

1. WHO. BCG Vaccine. Wkly Epidemiol Rec. 2004;79(4):27-48

2. WHO. Global tuberculosis report 2014 (WHO/HTM/ TB/2014.08) 2014

3. Smith KC, Orme IM, Starke JR. Vaccines [Internet]. Sixth Edit. Vaccines. Elsevier; 2013 [cited 2015 Jul 20]. 787-789 p. Available from: http://dx.doi.org/10.1016/B978-1-45570090-5.00040-9

4. Rodrigues LC, Diwan VK, Wheeler JG. Protective effect of BCG against tuberculous meningitis and miliary tuberculosis: a meta-analysis. Int J Epidemiol. 1993;22:1154-8

5. Romero Ml., Quiroz 0. PM. Dirección de Presupuestos 
División de Control de Gestión [Internet]. Final, Informe Inmunizaciones, Programa Nacional D E Salud, Ministerio D E. 2012. Available from: http://www.dipres.gob.cl/574/ articles-89691_doc_pdf.pdf

6. Nacional P, Transmisibles E. TUBERCULOSIS. 2014; Available from: http://epi.minsal.cl/epi/html/bolets/reportes/ tuberculosis/informe_tuberculosis.pdf

7. 7. Brennan MJ, Thole J. Tuberculosis vaccines: A strategic blueprint for the next decade. Tuberculosis (Edinb). 2012;92 Suppl 1:S6-13

8. Barreto ML, Pilger D, Pereira SM, Genser B, Cruz A, Cunha $S S$ et al. Causes of variation in BCG vaccine efficacy: examining evidence from the BCG REVAC cluster randomized trial to explore the masking and the blocking hypotheses. 2014 Jun 24;32:3759-64

9. Walker KB, Brennan MJ, Ho MM, Eskola J, Thiry G, Sadoff J, et al. The second Geneva Consensus: Recommendations for novel live TB vaccines. Vaccine. 2010;28:2259-70

10. Boom WH, Canaday DH, Fulton SA, Gehring AJ, Rojas RE, Torres M. Human immunity to M. tuberculosis: T cell subsets and antigen processing. Tuberculosis 2003;83:98-103

11. Gowthaman U, Mushtaq K, Tan AC, Rai PK, Jackson DC, Agrewala JN. Challenges and solutions for a rational vaccine design for TB-endemic regions. Crit Rev Microbiol 2014;7828:1-10

12. Jensen, KJ; Larsen N, Biering-Sørensen S, Andersen $A$, Eriksen HB, Monteiro I, Hougaard D, Aaby P, Netea MG, Flanagan KL, Benn CS. Heterologous Immunological Effects of Early BCG Vaccination in Low-Birth-Weight Infants in Guinea-Bissau: A Randomized-controlled Trial. J Infect Dis. 2015;211:956-67

13. Clothier HJ1, Hosking L, Crawford NW, Russell M, Easton ML, Quinn JA, Buttery JP. Bacillus Calmette-Guérin ( BCG ) Vaccine Adverse Events in Victoria, Australia : Analysis of Reports to an Enhanced Passive Surveillance System . Drug Saf 2015;38:79-86

14. Lakhar BB, Neonatal BCG and scar success. Indian Pediatr 1995;32:1323

15. 15. Ritz N, Tebruegge M, Camacho-Badilla K, Haeusler GM, Connell TG, Curtis N. To TST or not to TST: is tuberculin skin testing necessary before BCG immunisation in children? Vaccine 2012;14:1434-6

16. Sayyahfar S, Karimi A, Fahimzad A, Shamshiri AR. Comparison of Tuberculin Skin Test result and interferon gamma response to human PPD in BCG scar positive and negative children. J Epidemiol Glob Health 2014;4:45-50

17. Biering-Sørensen S, Jensen KJ, Aamand SH, Blok B, Andersen $A$, Monteiro I et al. Variation of growth in the production of the BCG vaccine and the association with the immune response. An observational study within a randomised trial. Vaccine 2015;33:2056-65

18. Mangtani P, Abubakar I, Ariti C, Beynon R, Pimpin L, Fine PEM et al. Protection by BCG vaccine against tuberculosis: a systematic review of randomized controlled trials. Clin Infect Dis $2014 ; 58: 470-80$

19. Ortqvist $\AA$, Blennow M, Carlsson R-M, Hanson LA, Lindberg a, Lindqvist $L$ et al. Vaccination of children--a systematic review. Acta Paediatr Suppl 2010;99:1-192
20. Fifteen year follow up of trial of BCG vaccines in south India for tuberculosis prevention. Tuberculosis Research Centre (ICMR), Chennai. [No authors listed].Indian J Med Res 1999; 110:56-69

21. Hart D'Arcy P, Sutherland I. BCG and vole bacillus vaccines in the prevention of tuberculosis in adolescence and early adult life. Final report to the Medical Research Council. $\mathrm{Br}$ Med J 1977;2:293-5

22. Clemens JD, Chuong JJ, Feinstein AR. The BCG controversy. A methodological and statistical reappraisal. JAMA. 1983 May 6;249:2362-9

23. Wilson ME, Fineberg H V, Colditz GA. Geographic latitude and the efficacy of bacillus Calmette-Guérin vaccine. Clin Infect Dis 1995;20:982-91

24. Colditz GA, Berkey CS, Mosteller F, Brewer TF, Wilson ME, Burdick E, Fineberg HV. The efficacy of bacillus CalmetteGuérin vaccination of newborns and infants in the prevention of tuberculosis: meta-analyses of the published literature. Pediatr1995;96:29-35

25. Subsecretaria de Salud Pública. Ordinario B27/N 2574 Lineaminetos de vacunación frente a situaciones especiales

26. Al-Kassimi FA, Al-Hajjaj MS, Al-Orainey IO, Bamgboye EA. Does the protective effect of neonatal BCG correlate with vaccine-induced tuberculin reaction? Am J Respir Crit Care Med 1995;152:1575-8

27. Citron KM, Lunn JA. Immunisation against infectious disease. BMJ 1998;297:1406

28. Toukam Tchakoute C, Hesseling AC, Kidzeru EB, Gamieldien $\mathrm{H}$, Passmore J-AS, Jones CE et al. Delaying BCG vaccination until 8 weeks of age results in robust BCG-specific $T$ cell responses in HIV-exposed infants. J Infect Dis 2015;211:338-46

29. Karp CL, Wilson CB, Stuart LM, Bill T, Foundation MG. Tuberculosis vaccines: barriers and prospects on the quest for a transformative tool. Immunol Rev 2015;363-81

30. Rodrigues LC, Mangtani P, Abubakar I. How does the level of BCG vaccine protection against tuberculosis fall over time? BMJ 2011;343:d5974 